\title{
A local fermion update algorithm for supersymmetric Yang-Mills quantum mechanics
}

\author{
Georg Bergner, Hang Liu and Urs Wenger* \\ Albert Einstein Center for Fundamental Physics \\ Institute for Theoretical Physics \\ University of Bern \\ Sidlerstrasse 5 \\ CH-3012 Bern \\ Switzerland \\ E-mail: bergneraitp.unibe.ch, liuaitp.unibe.ch, wengerditp.unibe.ch
}

We present a local fermion update algorithm for $\mathscr{N}=4$ supersymmetric Yang-Mills quantum mechanics which allows simulations in fixed canonical sectors. We discuss some aspects of the physics of this theory, including the appearance of flat directions in the bosonic potential and the metastabilities related to those. In particular, we show that the modulus of the bosonic fields diverges in some of the fermion sectors and for thermal boundary conditions.

34th annual International Symposium on Lattice Field Theory

24-30 July 2016

University of Southampton, UK

${ }^{*}$ Speaker. 


\section{Introduction}

These proceedings report on our ongoing effort to simulate supersymmetric Yang-Mills (SYM) gauge theories with gauge group $\mathrm{SU}(N)$. Our considerations start from the dimensional reduction of $\mathscr{N}=1 \mathrm{SYM}$ in $d=4$ dimensions with gauge group $\mathrm{SU}(N)$ which yields $\mathscr{N}=4 \mathrm{SYM}$ in one dimension, i.e. SYM quantum mechanics. In the process the three spatial components of the 4-dimensional gauge field become bosonic fields denoted by $X_{i}(t), i=1,2,3$, while the temporal component is denoted by $A(t)$. The fermionic degrees of freedom are represented by complex 2-component Grassmann fields $\bar{\psi}(t), \psi(t)$. All fields are in the adjoint representation and the complete action reads $[1,2]$

$$
S=\frac{1}{g^{2}} \int_{0}^{\beta} d t \operatorname{Tr}\left\{\left(D_{t} X_{i}\right)^{2}-\frac{1}{2}\left[X_{i}, X_{j}\right]^{2}+\bar{\psi} D_{t} \psi-\bar{\psi} \sigma_{i}\left[X_{i}, \psi\right]\right\}
$$

where $D_{t}=\partial_{t}-i[A(t), \cdot]$ is the covariant derivative and $\sigma_{i}$ are the Pauli matrices. The temporal extent $\beta$ of the system is discretized in the Euclidean direction using $L_{t}$ lattice points. In order to maintain gauge invariance of the discretized system, the continuum gauge field is replaced by gauge links $U(t)$ which are elements of the gauge group $\mathrm{SU}(N)$, and hence the lattice covariant derivative becomes $\hat{D}_{t} X_{i}(t)=U(t) X_{i}(t+1) U^{\dagger}(t)-X_{i}(t)$. The same difference operator also emerges for the fermions after inclusion of the Wilson term which breaks time reversal and hence charge conjugation symmetry. Supersymmetry is broken by the discretization as well. However, all symmetries are automatically restored in the continuum limit. For further reference, we include a chemical potential, which couples to the fermion number, and write out the fermion action as

$$
S_{F}=\frac{1}{2 g^{2}} \sum_{t=0}^{L_{t}-1}\left[-\bar{\psi}_{\alpha}^{a}(t) W_{\alpha \beta}^{a b}(t) e^{\mu} \psi_{\beta}^{b}(t+1)+\bar{\psi}_{\alpha}^{a}(t) \Phi_{\alpha \beta}^{a b}(t) \psi_{\beta}^{b}(t)\right] .
$$

The matrix $W$ connecting the fermion fields at different lattice sites reads

$$
W_{\alpha \beta}^{a b}(t)=2 \delta_{\alpha \beta} \cdot \operatorname{Tr}\left\{T^{a} U(t) T^{b} U^{\dagger}(t)\right\},
$$

where $T^{a}$ are the generators of the gauge group $\mathrm{SU}(N)$, and $\Phi$ is the $2\left(N^{2}-1\right) \times 2\left(N^{2}-1\right)$ Yukawa interaction matrix

$$
\Phi_{\alpha \beta}^{a b}(t)=\left(\sigma_{0}\right)_{\alpha \beta} \cdot \delta^{a b}-2\left(\sigma_{i}\right)_{\alpha \beta} \cdot \operatorname{Tr}\left\{T^{a}\left[X_{i}(t), T^{b}\right]\right\} .
$$

Here, $\sigma_{0}$ is the $2 \times 2$ unit matrix.

\section{Canonical formulation}

Usually, simulations are done using the grand canonical partition function

$$
Z_{p, a}=\int \mathscr{D} U \mathscr{D} X_{i} e^{-S_{B}\left[U, X_{i}\right]} \operatorname{det} \mathscr{D}_{p, a}\left[U, X_{i} ; \mu\right]
$$

where $S_{B}$ is the bosonic action and $\mathscr{D}_{p, a}$ the Wilson Dirac operator with periodic or antiperiodic fermion boundary conditions, respectively. Instead of fixing the chemical potential, one can also fix the fermion number $n_{f}$ and work with the canonical partition functions

$$
Z_{n_{f}}=\int \mathscr{D} U \mathscr{D} X_{i} e^{-S_{B}\left[U, X_{i}\right]} \operatorname{det} \mathscr{D}_{n_{f}}\left[U, X_{i}\right] .
$$


While the fugacity expansion relates the canonical and grand canonical partition functions and provides a relation between the corresponding determinants, the direct calculation of $\operatorname{det} \mathscr{D}_{n_{f}}$ constitutes a challenge for doing simulations directly in the canonical formulation. It turns out that the temporal reduction of the Wilson Dirac operator [3,4] based on Schur complement techniques is a crucial step for performing this task. The reduced determinant formula reads

$$
\operatorname{det} \mathscr{D}_{p, a}\left[U, X_{i} ; \mu\right]=\operatorname{det}\left[\mathscr{T} \mp e^{+\mu L_{t}}\right], \quad \mathscr{T}=\prod_{t=0}^{L_{t}-1}(\Phi(t) W(t))
$$

where the matrix $\mathscr{T}$ is of size $n_{f}^{\max } \times n_{f}^{\max }$ with $n_{f}^{\max }=2\left(N^{2}-1\right)$. Comparing this expression with the fugacity expansion and using some algebraic matrix identitites one obtains an explicit formula for the canonical determinants in terms of transfer matrices defined for fixed canonical sectors [3],

$$
\operatorname{det} \mathscr{D}_{n_{f}}\left[U, X_{i}\right]=\operatorname{Tr} \prod_{t=0}^{L_{t}-1}\left[T_{n_{f}}^{\Phi}(t) \cdot T_{n_{f}}^{W}(t)\right]=\operatorname{Tr} M_{n_{f}}(\mathscr{T})=\sum_{B} \operatorname{det} \mathscr{T}^{\mathcal{B} B}\left[U, X_{i}\right]
$$

Here, $T_{n_{f}}^{\Phi}(t) \cdot T_{n_{f}}^{W}(t)$ constitutes the transfer matrix at time $t$, which describes the transition probabilities of the $n=\left(\begin{array}{c}n_{f}^{\max } \\ n_{f}\end{array}\right)$ states in sector $n_{f}$, and $M_{n_{f}}(\cdot)$ denotes the matrix of minors of rank $n_{f}$, i.e., the index set $B \subseteq\left\{1,2, \ldots, 2\left(N^{2}-1\right)\right\}$ is of size $n_{f}[3]$.

\section{Simulation algorithms}

There are several possibilitites to simulate the canonical partition functions based on eq.(2.1). Results from simulating directly the transfer matrices will be described elsewhere, while here we concentrate on a simulation strategy based on the sum of the principal minors,

$$
Z_{n_{f}}=\sum_{B} \int \mathscr{D} U \mathscr{D} X_{i} e^{-S\left[U, X_{i}\right]} \operatorname{det} \mathscr{T}^{\mathcal{B} B}\left[U, X_{i}\right]
$$

Since the number $n$ of principal minors of order $n_{f} \sim n_{f}^{\max } / 2$ grows factorially with the size $n_{f}^{\max }$ of $\mathscr{T}$, an exact evaluation of the canonical determinant is impractical or even impossible. Instead, we employ an efficient stochastic evaluation of the sum of the principal minors and treat the summation index set $B$ as an additional degree of freedom of the system. Thereby, the index set $B$ is dynamically updated using a standard Metropolis algorithm. Starting from the index set $B$ a new random set $B^{\prime}$ is proposed using Fisher-Yates reshuffling and the transition $B \rightarrow B^{\prime}$ is accepted with the probability $p_{B \rightarrow B^{\prime}}=\min \left[1, A_{B \rightarrow B^{\prime}}\right]$ with

$$
A_{B \rightarrow B^{\prime}}=\left|\frac{\operatorname{det} \mathscr{T}\left[U, X_{i}\right]^{B^{\prime} \mathcal{B}^{\prime}}}{\operatorname{det} \mathscr{T}\left[U, X_{i}\right]^{\not B k}}\right| .
$$

Then, the remaining fields are updated keeping the index set fixed. In order for this whole process to be practical, one needs an efficient calculation, or update, of the full matrix $\mathscr{T}$. We are using a binary tree data structure to store intermediate products, such that only $O\left(\ln L_{t}\right)$ matrix multiplications are necessary instead of $O\left(L_{t}\right)$. 

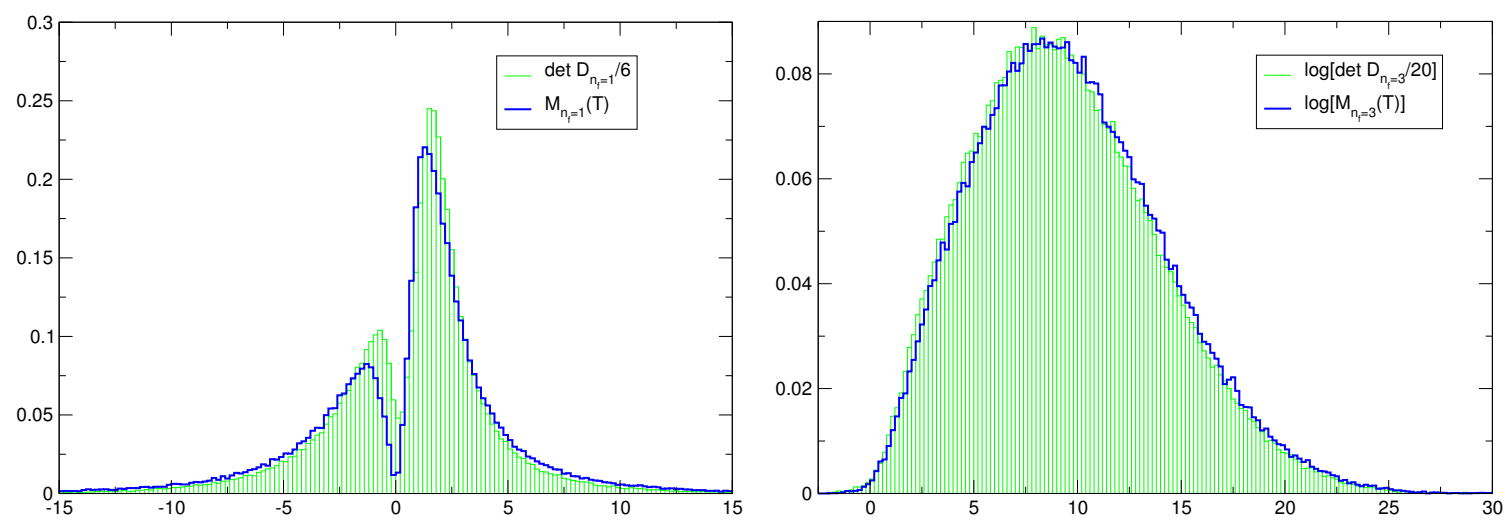

Figure 1: Distributions of the principal minors $M_{n_{f}}(\mathscr{T})$ from the stochastic evaluation of the trace and the corresponding canonical determinant for SU(2), $L_{t}=24$ at $\beta=1.2$ in sector $n_{f}=1$ (left plot) and $n_{f}=3$ (right plot).

The prinicipal minors need not be positive, but can have negative signs, in which case reweighting with the reweighting factors $R_{n_{f}}=\operatorname{sign}\left(M_{n_{f}}\right)$ is required. ${ }^{1}$ As illustrated in the left plot of Fig. 1, the Metropolis algorithm has no difficulty to tunnel between the sectors of the configuration space which yield negative and positive contributions, but in fact samples both contributions very efficiently. This is due to the fact that the Metropolis algorithm allows the proposal and occasional acceptance of large changes of the fields on the one hand, and sign changes in the determinant from updating the index set on the other hand. We further note that the negative contributions are absolutely necessary for reweighting the configurations to different fermion number sectors, but they are not frequent enough to generate a severe sign problem. In fact, negative contributions do not occur in all sectors. For SU(2) for example, contributions in sectors $n_{f}=0,6$ are strictly positive, while sectors $n_{f}=1,5$ have significant negative contributions. In sectors $n_{f}=2,3,4$ the negative contributions are negligible, cf. right plot of Fig. 1.

Also shown in Fig. 1 are the distributions of the principal minors $M_{n_{f}}(\mathscr{T})=\operatorname{det} \mathscr{T}[U, X]^{k B}$ as they occur in the stochastic evaluation of the sum, i.e. the trace $\operatorname{Tr} M_{n_{f}}$, in comparison with the distribution of the corresponding full canonical determinant divided by the number of principal minors in the given sector. We see that the principal minors follow the full determinant very closely and evolve the system very similarly, but their evaluation is by a factor $n$ faster. While for SU(2) this factor is maximally 20, for SU(3) the maximal gain is already 12870 .

One peculiarity of the model considered here is the fact that it possesses so-called flat directions (moduli space) along which $\left[X_{i}, X_{j}\right]=0$. As a consequence, the system may suffer from $X_{i}$ running away and $X^{2} \equiv 1 / L_{t} \sum_{t=0}^{L_{t}-1} \operatorname{Tr}\left\{X_{i}(t) X_{i}(t)\right\}$ becoming arbitrarily large. While one can regularize the divergence with the deformation $m^{2} X^{2}$, we observe that metastable states along the flat directions may be introduced due to an interplay between lattice artefacts and the deformation, and the simulation consequently suffers from critical slowing down. Essentially, in those metastable states fluctuations orthogonal to the flat direction are highly suppressed compared to those along the flat direction, hence the standard Metropolis becomes highly inefficient. A solution to this

\footnotetext{
${ }^{1}$ Reweighting between different fermion sectors is also possible and turns out to be reliable in certain cases, cf. Ref. [5].
} 

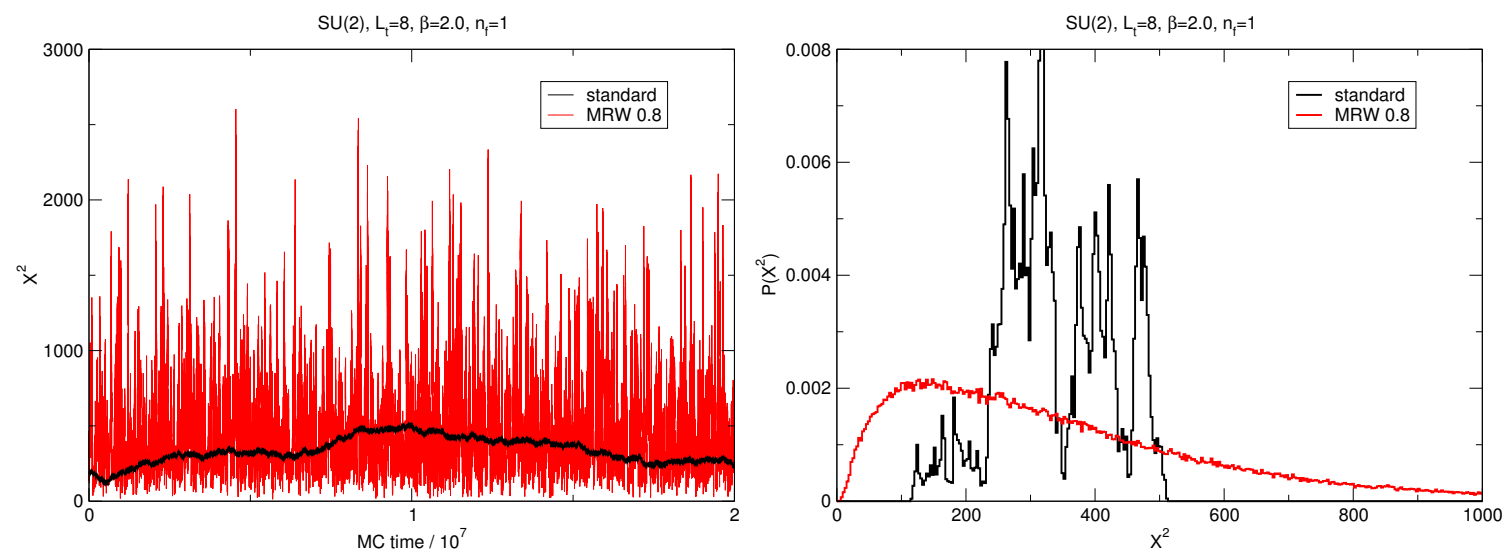

Figure 2: MC time history (left plot) and the corresponding distribution (right plot) of the moduli $X^{2}$ for $\mathrm{SU}(2)$ on a $L_{t}=8$ lattice at $\beta=2.0$ in the $n_{f}=1$ sector, once with the standard Metropolis algorithm and once with the multiplicative random walk (MRW) algorithm.

problem is provided by the multiplicative random walk (MRW) update algorithm which updates the bosonic fields $X$ collectively by rescaling them by a global random factor $R$. In order to fulfill detailed balance, the acceptance probability has to be chosen with care. The procedure is illustrated in Fig. 2 where we show the MC time history (left plot) and the corresponding distribution (right plot) of the moduli $X^{2}$ for a system which is stuck in a flat direction (for $\mathrm{SU}(2)$ on a $L_{t}=8$ lattice at $\beta=2.0$ in the $n_{f}=1$ sector). With the standard Metropolis algorithm $X^{2}$ moves very slowly and the huge autocorrelations are reflected in the completely unreliable distribution of $X^{2}$. In contrast, the MRW algorithm samples $X^{2}$ very efficiently and produces a smooth distribution which in turn allows a reliable extraction of $\left\langle X^{2}\right\rangle$.

\section{Results}

We first investigate the behaviour of the stable and metastable phases as a function of the regulator mass $m$ in the limit $m \rightarrow 0$. In order to do so, we prepare the system with a starting configuration consisting of small fluctuations around $X=0$ (small random field) on the one hand and with an added large component along a flat direction on the other hand. As can be seen from Fig. 3, for sufficiently large $m$ a system prepared in a phase along the flat direction always tunnels back into the phase exhibiting small fluctuations only, while for $L_{t} m \lesssim 0.07$ the tunneling barrier is too large and hence the system becomes metastable. The minimum of the metastable phase moves to infinity and $\left\langle X^{2}\right\rangle$ diverges in the limit $m \rightarrow 0$ as $1 /\left(L_{t} m\right)^{2}$ to leading order. Let us emphasize that the MRW algorithm is crucial to obtain reliable results in the metastable phase. In contrast, simulations starting from small random field configurations seem stable and $\left\langle X^{2}\right\rangle$ is well behaved in the limit $m \rightarrow 0$. In fact, for sufficiently large $L_{t}$ even simulations at $m=0$ are possible.

Having clarified the role and fate of the metastable phase, we can now investigate in detail the regulator dependence of the persisting stable phase involving small fluctuations of $X$. In Fig. 4 we show the results for $\left\langle X^{2}\right\rangle$ as a function of $m$ from simulations of $\mathrm{SU}(2)$ at $\beta=0.5$ for a range of lattice extents $L_{t}$ in some fermion number sectors, as well as for periodic and antiperiodic fermionic boundary conditions. Here, $\left\langle X^{2}\right\rangle$ is rescaled by $L_{t}$, and the continuum limit corresponds to $L_{t} \rightarrow \infty$ 

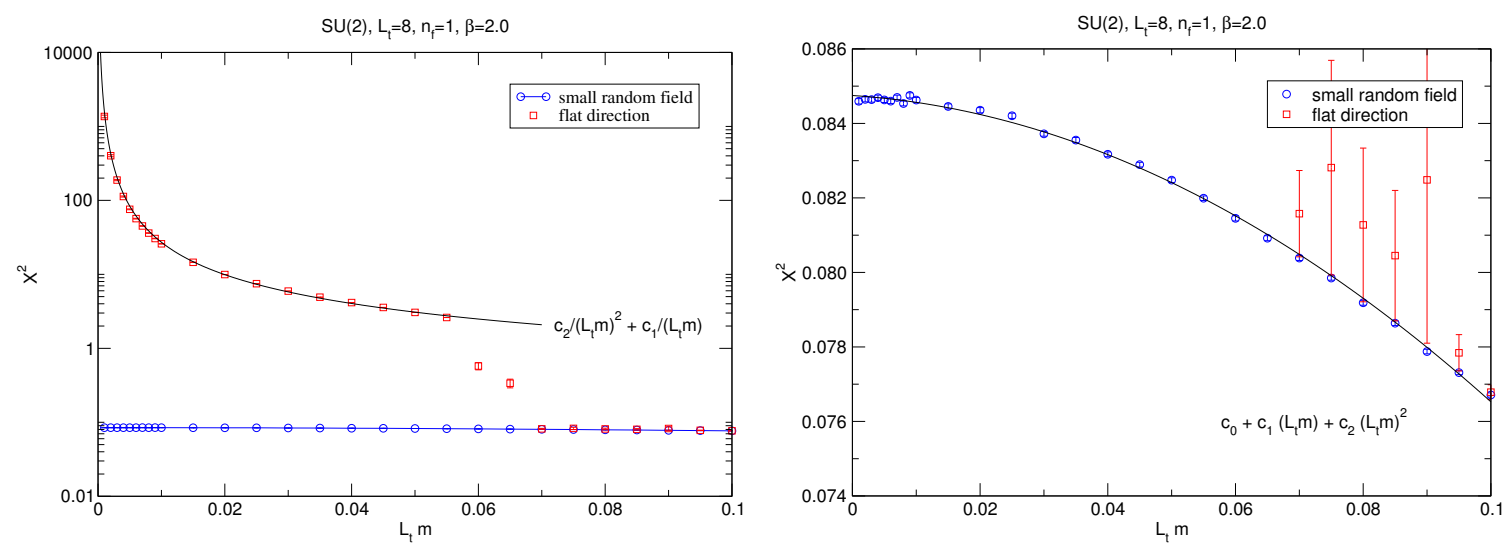

Figure 3: The expectation value of the moduli $X^{2}$ as a function of the regulator mass for SU(2) on a $L_{t}=8$ lattice at $\beta=2.0$ in the $n_{f}=1$ sector, once starting from a configuration consisting of small random fluctuations, and once with a large added component along a flat direction. The right plot is a zoom to the small region of $X^{2}$.

at fixed $L_{t} m$. Let us first discuss the lattice artefacts. For large $m$ we observe universal scaling behaviour independent of the lattice spacing $a=\beta / L_{t}$. In this regime, lattice artefacts become very small and the degeneracy between the charge conjugated fermion number sectors is restored. When the regulator is removed, lattice artefacts become large and the effect from the explicitly broken charge conjugation symmetry $\mathscr{C}$ becomes evident. Only for the sectors $n_{f}=2$ and 4 the $\mathscr{C}$-symmetry seems to remain exact at a finite lattice spacing. In sector $n_{f}=6$ the lattice artefacts are tiny. The latter sector corresponds to the quenched approximation, hence the leading artefacts are expected to be $O\left(a^{2}\right)$ in contrast to the other sectors where they are $O(a)$.

Next we discuss the behaviour of $\left\langle X^{2}\right\rangle$ at $m=0$ in the continuum limit. First we note that simulations at $m=0$ at finite $L_{t}$ seem to be possible - apparently, the simulations are stabilized by lattice artefacts. Towards the continuum, the fermion sectors with $n_{f}=2,3,4$ qualitatively show a different behaviour than the sectors with $n_{f}=0,1,5,6$. For the latter, $\left\langle X^{2}\right\rangle$ approaches a finite value in the continuum limit, while for the former, $\left\langle X^{2}\right\rangle$ appears to diverge. Note that this divergence has nothing to do with the divergence in the metastable phase discussed before, but is expected due to zero energy states and the spectrum becoming continuous in those sectors [6,7]. Similarly, $\left\langle X^{2}\right\rangle$ is finite in the system with periodic b.c., because the diverging contributions from sectors $n_{f}=2,3,4$ cancel each other, while this does not happen for the system with antiperiodic b.c..

To summarize, we presented an efficient local fermion algorithm which allows simulations in fixed canonical sectors in a completely controlled way. Hence, our approach is an alternative to other numerical efforts investigating supersymmetric Yang-Mills quantum mechanics, such as $[8,9]$.

\section{References}

[1] S. Catterall and T. Wiseman, Towards lattice simulation of the gauge theory duals to black holes and hot strings, JHEP 0712 (2007) 104, [0 706 . 3518]. 

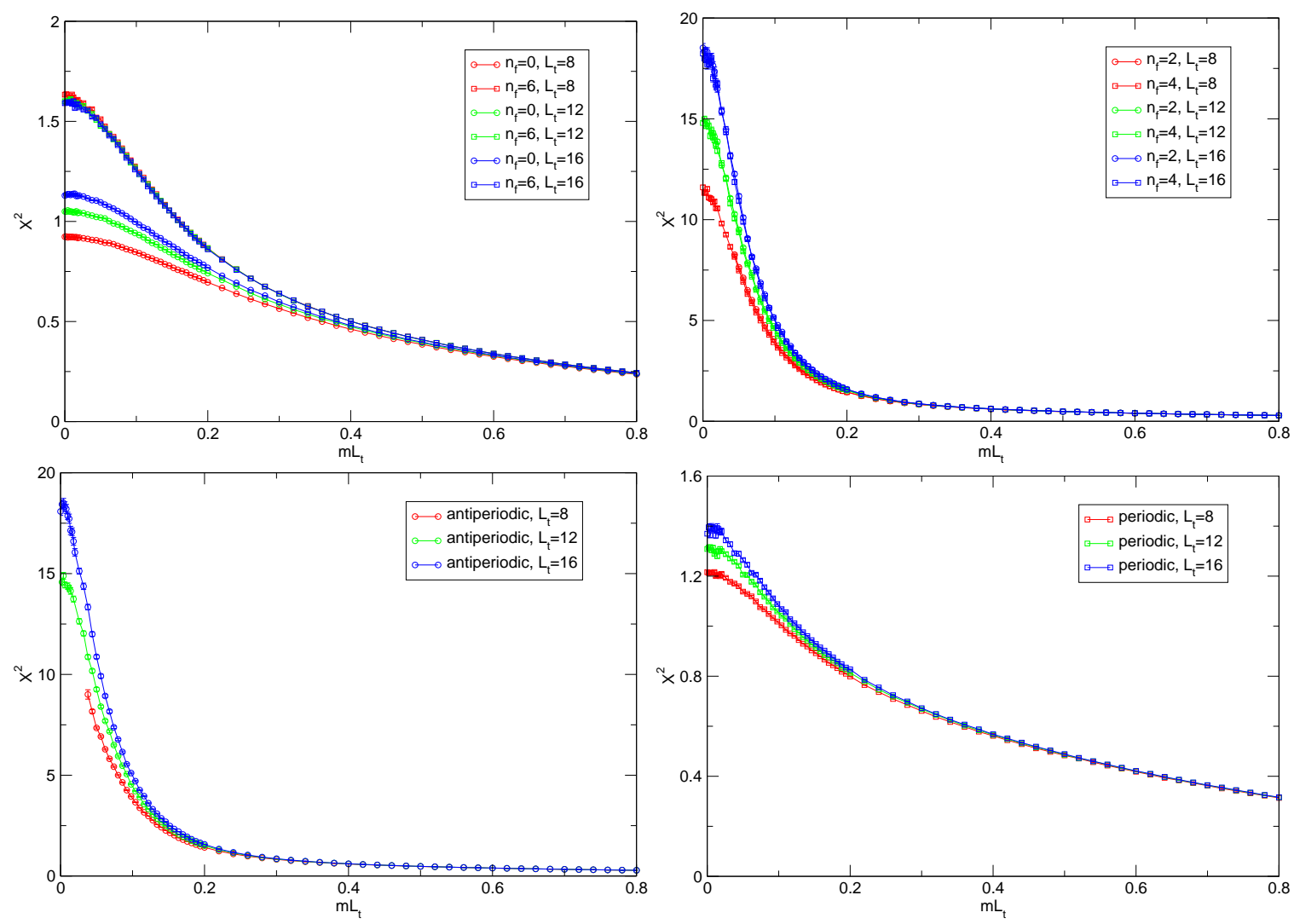

Figure 4: The expectation value of the moduli $X^{2}=\left\langle\operatorname{Tr} X_{i}^{2}\right\rangle$ as a function of the regulator mass for SU(2) and various lattice extents $L_{t}=8,12,16$ at $\beta=0.5$ in the sectors with $n_{f}=0, \ldots, 6$ and for periodic and antiperiodic boundary conditions.

[2] S. Catterall and T. Wiseman, Black hole thermodynamics from simulations of lattice Yang-Mills theory, Phys.Rev. D78 (2008) 041502, [0 803 . 4273].

[3] K. Steinhauer and U. Wenger, Loop formulation of supersymmetric Yang-Mills quantum mechanics, JHEP 12 (2014) 044, [1410.0235].

[4] A. Alexandru and U. Wenger, QCD at non-zero density and canonical partition functions with Wilson fermions, Phys. Rev. D83 (2011) 034502, [1009.2197].

[5] G. Bergner, H. Liu and U. Wenger, Canonical simulations of supersymmetric SU(N) Yang-Mills quantum mechanics, PoS LATTICE2015 (2016) 241, [1509. 01446 ].

[6] J. Wosiek, Spectra of supersymmetric Yang-Mills quantum mechanics, Nucl.Phys. B644 (2002) 85-112, [hep-th/0203116].

[7] M. Campostrini and J. Wosiek, High precision study of the structure of D=4 supersymmetric Yang-Mills quantum mechanics, Nucl. Phys. B703 (2004) 454-498, [hep-th / 0407021 ].

[8] Z. Ambroziński and P. Korcyl, Numerical investigations of Supersymmetric Yang-Mills Quantum Mechanics with 4 supercharges, PoS LATTICE2014 (2015) 253, [1411.6904].

[9] E. Berkowitz, E. Rinaldi, M. Hanada, G. Ishiki, S. Shimasaki and P. Vranas, Precision lattice test of the gauge/gravity duality at large-N, Phys. Rev. D94 (2016) 094501, [1606. 04951$].$ 